\title{
Mechanisms of odor-tracking: multiple sensors for enhanced perception and behavior
}

\author{
Alex Gomez-Marin ${ }^{1+}$, Brian J. Duistermars ${ }^{2+}$, Mark A. Frye ${ }^{2 *}$ and Matthieu Louis ${ }^{1 *}$ \\ EMBL-CRG Systems Biology Unit, Centre for Genomic Regulation, Universitat Pompeu Fabra, Barcelona, Spain \\ 2 Howard Hughes Medical Institute, Department of Physiological Science, University of California, Los Angeles, CA, USA
}

Edited by:

Hernan Lopez-Schier,

Centre de Regulació Genòmica - PRBB,

Spain

Reviewed by:

Bertram Gerber,

Universität Würzburg, Germany

Marcus Stensmyr,

Max Planck Institute for Chemical

Ecology, Germany

${ }^{*}$ Correspondence:

Mark A. Frye, Howard Hughes Medical Institute, Department of Physiological

Science, University of California, Los

Angeles, CA 90095, USA.

e-mail:frye@ucla.edu; Matthieu Louis, EMBL-CRG Systems Biology Unit,

Centre for Genomic Regulation,

Universitat Pompeu Fabra, Dr. Aiguader

88, Barcelona 08003, Spain.

e-mail:mlouis@crg.es

${ }^{\dagger}$ Alex Gomez-Marin and Brian J.

Duistermars have contributed equally

to this work.
Early in evolution, the ability to sense and respond to changing environments must have provided a critical survival advantage to living organisms. From bacteria and worms to flies and vertebrates, sophisticated mechanisms have evolved to enhance odor detection and localization. Here, we review several modes of chemotaxis. We further consider the relevance of a striking and recurrent motif in the organization of invertebrate and vertebrate sensory systems, namely the existence of two symmetrical olfactory sensors. By combining our current knowledge about the olfactory circuits of larval and adult Drosophila, we examine the molecular and neural mechanisms underlying robust olfactory perception and extend these analyses to recent behavioral studies addressing the relevance and function of bilateral olfactory input for gradient detection. Finally, using a comparative theoretical approach based on Braitenberg's vehicles, we speculate about the relationships between anatomy, circuit architecture and stereotypical orientation behaviors.

Keywords: drosophila melanogaster, olfaction, bilateral, chemotaxis, orientation behavior, sensory perception

\section{INTRODUCTION MODES OF CHEMOTAXIS}

To survive and prosper, motile organisms must extract relevant cues from their environment, integrate them into internal representations, and react adequately to each contingency. Encoding and processing chemical signals is essential to locate food, flee danger and engage in proper social interactions. The prototype of unicellular chemotaxis is illustrated by the bacterium Escherichia coli (Berg, 2004). Its navigational performance operates effectively within the severe physical constraints of its aqueous environment. This micron-scale organism uses flagella to propel itself at a speed equivalent to 20 times its body length per second despite high viscous drag. Due to thermal fluctuations, E. coli undergoes random perturbations in heading which prevent it from maintaining linear motion for more than a few seconds. Furthermore, its accuracy in determining the concentration of attractants is limited by fluctuations in the number of odorant molecules in the cell's surroundings (Berg and Purcell, 1977). Despite these physical facts, E. coli excels at tracking sugar sources while keeping away from poisonous environments. According to Berg and Purcell, the chemotactic sensitivity of E. coli approaches that of an "optimum design".

In pioneering studies, Julius Adler established causal connections between changes in chemical concentration in the environment, methylation state of chemoreceptors and direction of flagellar rotation (Adler, 1969). By tracking trajectories of E. coli at high resolution, Berg and Brown showed that bacterial swims consist of two modes of motion: straight runs lasting about a second, and brief stops lasting a tenth of a second during which the orientation of the cell is randomized (Berg and Brown, 1972). The authors called these two modes of motion "runs" and "tumbles" (or "twiddles") (Figure 1A). Fluorescence microscopy further revealed that counterclockwise rotation of the flagella creates a compact bundle that propels the bacterium forward during a "run". When one (or several) flagellum rotates clockwise, the bundle is disrupted, propulsion stops and, consequently, forward motion ceases as at low Reynolds numbers viscous drag overtakes inertia (Purcell, 1977).

The centerpiece of the bacterial chemotactic algorithm is the modulation of the tumbling frequency as a function of the concentration $(C)$ of relevant chemicals surrounding the cell. If by chance $E$. coli ascends an attractive gradient $(\Delta C / \Delta t>0)$, tumbling events are less frequent. When the cell travels down the gradient $(\Delta C / \Delta t<0)$, its motion is indistinguishable from that in isotropic environments. Hence, E. coli can be viewed as an "optimist" organism: when conditions worsen, it tumbles looking for improvements. When conditions improve, it avoids making any change. It is worth emphasizing that $E$. coli is unable to "choose" its direction of movement; it modifies its orientation randomly. Only the probability of transition between the two modes of motion is controlled in a stimulus-dependent manner. Despite the fact that turns are not continuously aligned with the direction of the gradient, 


\section{A Escherichia coli}
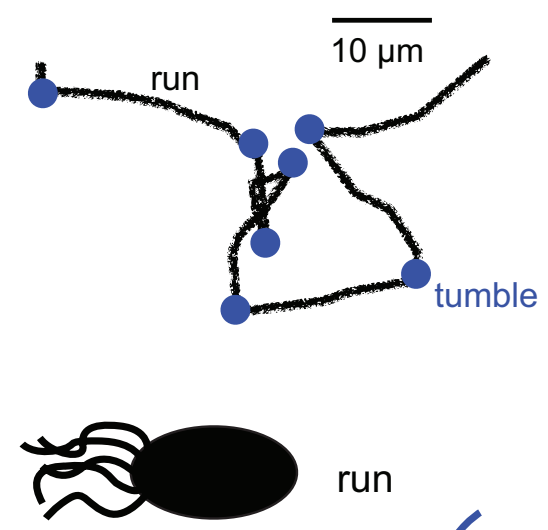

run

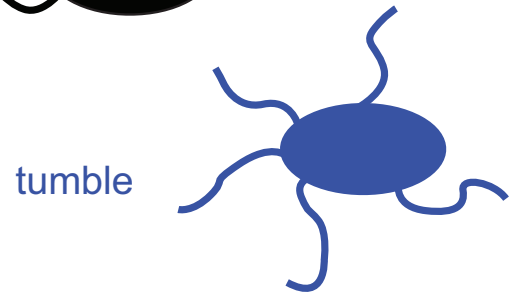

B
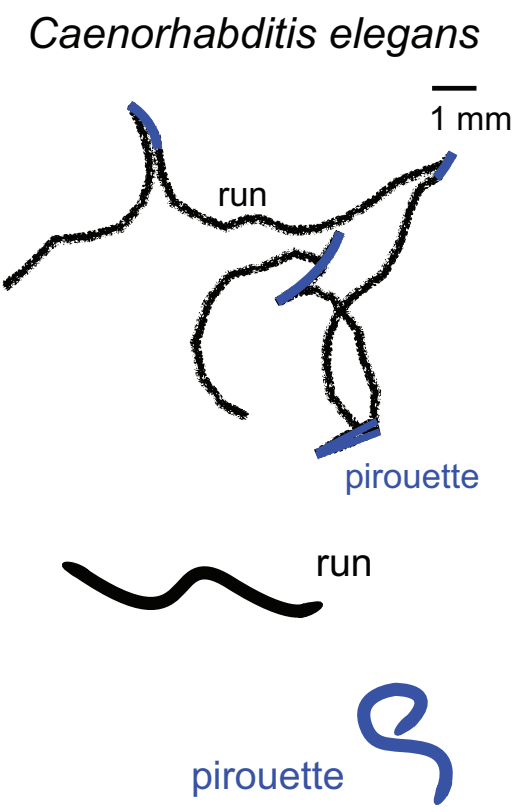

FIGURE 1 | Main orientation strategies underpinning chemotaxis in E. coli (A) and C. elegans (B). Trajectory segmentation models for E. coli and C. elegans (paths adapted from Berg and Brown, 1972,

and from Pierce-Shimomura et al., 1999) reveal that the probability of switching between "run" and "turn" modes is modulated by the time derivative of the concentration experienced by the organism. This simple mechanism leads to indirect navigation (kinesis) towards attractants and away from repellents. this strategy suffices to generate robust chemotaxis. Such indirect orientation responses are generically called kinesis (Fraenkel and Gunn, 1961).

A similar chemotactic strategy is observed in the nematode Caenorhabditis elegans where trajectories can be segmented into relatively straight runs and bouts of sharp turns termed pirouettes (Pierce-Shimomura et al., 1999) (Figure 1B). The likelihood of initiating a pirouette is high when the worm is heading down an attractive gradient $(\Delta C / \Delta t<0)$. Conversely, pirouettes are suppressed when the worm is heading up the gradient $(\Delta C / \Delta t>0)$. Similar to $E$. coli, sequential runs are biased in the direction of the highest odor concentration by continuously suppressing reorientations while ascending an attractive gradient. One notable difference exists between bacterial and nematode chemotaxis. While bacterial tumbles produce a random change in the direction of locomotion, pirouettes correct orientation by sequential error compensation. This last observation led Pierce-Shimomura et al. (1999) to hypothesis that chemotaxis by $C$. elegans represents an intermediate stage between biased random locomotion and the continuous alignment of heading relative to an odor gradient.

Although the fitness of bacteria and nematodes attest that chemotaxis can be achieved efficiently without extracting directional information from the stimulus, one may wonder whether animals with higher cognitive abilities have developed improved strategies based on the integration of directional inputs (taxis). Part of the answer lies in the existence of a common motif in the organization of most olfactory systems: "noses" are usually formed by a pair of symmetrical sensory organs. There is compelling evidence supporting the idea that bilateral olfactory input significantly enhances the accuracy of scent-tracking in arthropods and mammals (Porter et al., 2007; Louis et al., 2008a). To examine the extent to which the stereo sampling of odor cues confers a sensory advantage, we first consider this question in the context of honeybees, ants, rats and humans.

\section{EVIDENCE FOR THE USE OF STEREO OLFACTION IN INSECTS AND VERTEBRATES}

Insects with spatially distributed sensory organs could, in theory, sample the environment by comparing the inputs transmitted by each antenna or by moving their sensors in space to evaluate the stimulus field at different locations. Several decades ago, Martin demonstrated that walking honeybees make use of both mechanisms (Martin, 1965). As long as a concentration difference is perceived between the left and the right antenna, bees turn towards the side detecting the highest intensity. Martin showed that, except from occasional and slight zigzagging, orientation was scarcely impaired in unilaterally antennectomized bees with a single unrestrained antenna. Also, crossing and fixing the left and the right antennae led to spatial confusion in orientation tasks as most bees turned towards the odorless branch of a Y-maze. When the tips of crossed antennae were placed closer than $2 \mathrm{~mm}$, Martin observed a transition from osmotropotaxis (simultaneous comparisons between left and right antennae) to klinotaxis (successive sampling by lateral head movements). In this case, the slope of the odor gradient determines whether walking bees operate according to osmotropotaxis or klinotaxis.

Stereo chemosensation is also exemplified in other insects. The black ants, Lasius fuliginosus, follow chemical trails based on an osmotropotactic mechanism where concentration differences are 
measured between the left and right antennae (Schone, 1984). While walking along a pheromone trail, ants tend to maintain the area of maximum concentration between the tips of their antennae. Chemotaxis results from sequential turns towards the antenna experiencing the highest excitation. Although stereo input is necessary for smooth orientation, unilaterally antennectomized ants are capable of following trails by abruptly correcting their course after large drifts from the trail center (Hangartner, 1967). Recently, stereo olfaction has been shown to play a critical role in the homing behavior of another species of ants, Cataglyphis fortis, which operate at temperatures too high for robust pheromone trailing (Steck et al., 2009, 2010). After foraging expeditions, nest re-localization by these ants can be guided by stereo olfactory landmarks which are compared to the nest olfactory layout memorized before departure (Steck et al., 2010).

In vertebrates, recent evidence supports the notion that stereo cues are relevant to locate odor sources in space. Behavioral experiments conducted in rats have shown that bilateral sniffing is necessary for accurate odor tracking (Rajan et al., 2006). Rats can gather spatial information about the stimulus direction in just one sniff, a time window smaller than $100 \mathrm{~ms}$ during which detection of inter-nasal timing differences must be taking place. Similarly, human scent-tracking is significantly improved by internostril comparisons (Porter et al., 2007) further highlighting the importance of bilateral olfactory input for the localization of odor cues.

Our knowledge about the nervous systems of rats and humans is still too fragmentary to explain the previous observations in terms of circuit computation. In contrast, the last two decades have witnessed significant progress made towards a comprehensive characterization of the fruit fly olfactory system (Stocker, 2001; Couto et al., 2005; Fishilevich and Vosshall, 2005; Vosshall and Stocker, 2007; Laissue and Vosshall, 2008; Masse et al., 2009). Anatomical and functional studies have revealed the high degree of structural similarity between the fly and vertebrate olfactory circuits (Wilson and Mainen, 2006). Accordingly, D. melanogaster has emerged as a premier model organism to study the molecular and neural basis of sensory perception and behavior. Larvae and adult flies demonstrate a rich repertoire of odor-driven behaviors controlled by a brain amenable to genetic manipulations and electrophysiological inspections. Thus, flies offer a convenient entry point to examine the neural circuit computation underlying complex behavior.

\section{THE FRUIT FLY OLFACTORY SYSTEM: STRUCTURE AND FUNCTION}

The lifecycle of D. melanogaster comprises four stages: egg, larva, pupa, and adult. During the two motile cycles, larva and adult, flies execute stereotypical odor-evoked behaviors. The larval and the adult olfactory systems share many of the same principles of organization but the two systems differ dramatically in the number of neurons they involve. At the larval stage, flies centuplicate their weight in about 6 days. Larvae are motivated by a single unique objective: localizing and staying on or near food sources. Olfaction seems to play an important role in this process (Asahina et al., 2008). Early on, many groups have demonstrated that larvae respond to a wide range of volatile odors (Aceves-Pina and Quinn, 1979; Rodrigues,
1980; Monte et al., 1989; Cobb et al., 1992). Over the past two decades, the peripheral olfactory system of the larva has emerged as a simplified version of its adult counterpart (reviewed in Gerber and Stocker, 2007).

The larval brain consists of two hemispheres, each composed of approximately 10,000 neurons. The larval "nose" is composed of two bilaterally symmetric olfactory organs, called dorsal organs (DOs). Histology and extracellular recordings have indicated that the DOs are the primary and very likely unique olfactory organs of larvae (Welsh, 1937; Heimbeck et al., 1999; Oppliger et al., 2000; Larsson et al., 2004). Each DO contains a central dome (5- $\mu \mathrm{m}$ diameter) protruding from the tip of the head (Figure 2A) (Cobb, 1999). Electron microscopy has revealed that the dome is perforated by thousands of pores permitting the diffusion of volatile odors between the external environment and the interior of the dome. This enables the binding of odorant molecules to odorant receptors (ORs) which reside in the dendritic arbors of olfactory receptor neurons (ORNs) (Figure 2A, inset). Whereas it remains unclear whether larvae are capable of long-range odor detection, olfaction provides a clear survival advantage under conditions of food shortage (Asahina et al., 2008). Presumably, larvae use their keen sense of smell to stay confined in ripe parts of decaying fruits, while avoiding high ethanol concentrations due to advanced fermentation. Also, several lines of evidence suggest that larvae use a primitive form of chemical communication to forage in groups of the same species (Wu et al., 2003; Beltrami et al., 2010).

At the adult stage, the Drosophila brain contains $\sim 100,000$ neurons. Adult flies are equipped with two pairs of olfactory organs: the third antennal segments and the maxillary palps (Figure 2B) (Shanbhag et al., 2000). These organs are covered with several classes of sclerotized olfactory bristles, or sensilla, which house the dendrites of ORNs (Figure 2B, inset) (Vosshall et al., 1999; Stocker, 2001). Similar to larvae, odorant molecules from the external environment pass through the perforated sensillar cuticle, diffuse into endolymph and bind to ORs residing within the dendritic membranes of ORNs (Figure 2B, inset).

Adult flies display a rich repertoire of behaviors driven, or modulated, by olfactory input. Odor cues modulate walking and flight behaviors (Cho et al., 2004; Frye and Dickinson, 2004; Budick and Dickinson, 2006; Reynolds and Frye, 2007; Duistermars and Frye, 2008; Kreher et al., 2008; Serway et al., 2009) and adults rely on olfaction to identify food sources and acceptable sites for oviposition (Joseph et al., 2009). Also, pheromones orchestrate social communication between individuals by signaling gender and mating states (Ejima et al., 2007; Datta et al., 2008; Miyamoto and Amrein, 2008).

\section{ODORANT RECEPTORS AND FIRST-ORDER OLFACTORY RECEPTOR NEURONS}

In both larvae and adults, olfactory perception begins when odorant molecules bind to odorant receptors (ORs) (Figure 2C) in olfactory receptor neuron (ORN) membranes (Buck and Axel, 1991). For agonistic odors, this interaction occurs in less than $1 \mathrm{~ms}$ (Bhandawat et al., 2005). Through ionotropic and/or metabotropic signaling (Sato et al., 2008; Wicher et al., 2008), the binding of agonists to ORs lead to the opening of OR-associated ion channels and a subsequent depolarization of ORNs. ORN action potentials are 


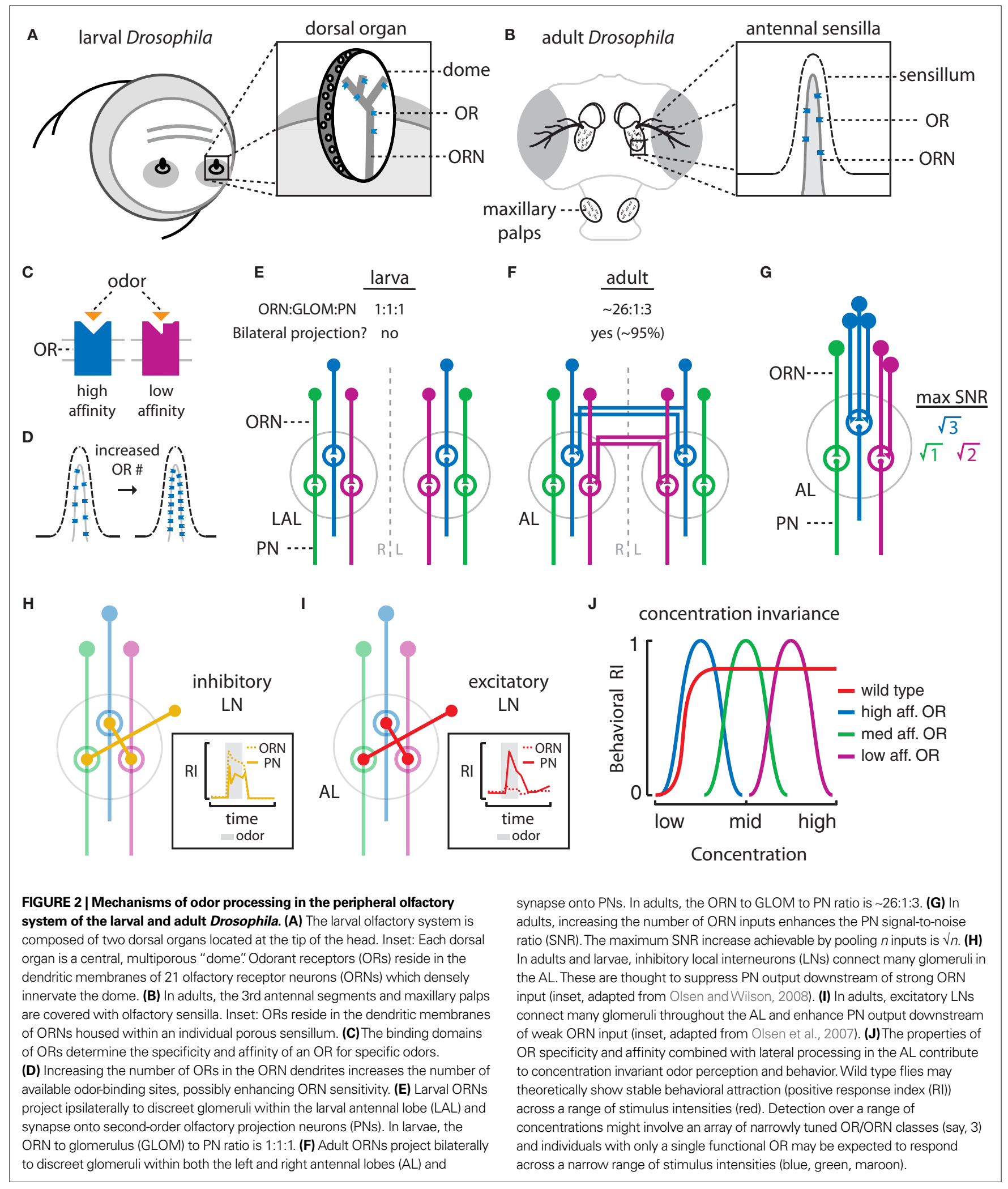

elicited when the combined activity of ORs across the dendrites bring the ORN to activation threshold (Bhandawat et al., 2005). ORNs can vary in the total number of ORs expressed within a single ORN (Kopp et al., 2008). Increasing the total number of ORs and, as a result, the number of available olfactory binding sites, may enhance odor sensitivity (Figure 2D) (Dekker et al., 2006). Additionally, OR specificity, ligand affinity, and in turn, ORN specificity depends on the amino acid sequences of the odor-binding 
pockets of OR proteins (Figure 2C) (Afshar et al., 1998; Abaffy et al., 2007). Mutations in the primary amino acid sequence of a given OR can have significant effects on the sensitivity to a specific odor (Dekker et al., 2006; Keller et al., 2007).

The genome of $D$. melanogaster contains $\sim 62$ conventional ORs (Vosshall and Stocker, 2007). Larvae express $\sim 25$ ORs in 21 distinct ORNs while adults express $\sim 45$ ORs in more than 38 ORN classes (Couto et al., 2005). Only 12 of the 45 ORs present at the adult stage are also expressed in larvae (Vosshall and Stocker, 2007). A combination of RNA in situ hybridization, RT-PCR and Gal4 driver analyses (Couto et al., 2005; Fishilevich et al., 2005; Kreher et al., 2005) has revealed that larval and adult ORNs express one, or occasionally two, conventional ORs together with an atypical co-receptor OR83b. OR83b is believed to form multimeric complexes with the conventional ORs; its expression is necessary for the proper trafficking of all ORs to the dendrites and for their function as ligand-gated ion channels (Benton et al., 2006; Pellegrino and Nakagawa, 2009). In loss-of-function experiments, it has been found that odor-evoked behaviors to a large class of alcohols, aldehydes, ketones and aromatic molecules are abolished in OR83b null mutants (Larsson et al., 2004). Recently, a novel family of ionotropic glutamate receptors (IRs) has been found to be expressed in adult sensory neurons housed in coeloconic sensilla (Benton et al., 2009). In adult flies, IRs have been implicated in the detection of amines, in particular ammonia, acids and specific aldehydes, but their function as chemosensory receptors is still poorly documented.

The odor response spectra of most ORNs have been characterized in situ in the adult empty neuron system (Hallem et al., 2004; Kreher et al., 2005) as well as by calcium imaging (Asahina et al., 2009), in vivo electrophysiology (Hoare et al., 2008), and in behavioral assays (Fishilevich et al., 2005). A given ORN can be excited or inhibited by distinct ligands (Hallem and Carlson, 2006; Kreher et al., 2008). Some ORNs are narrowly tuned and activated by only a few odors (e.g. ORNs expressing OR82a and OR94b), whereas others are broadly tuned and respond to many odors (e.g. ORNs expressing OR42a and OR85c). This indicates that the olfactory system of D. melanogaster is composed of 21 (larva) and over 38 (adult) independent information channels with overlapping and unique tuning profiles. Hence, it is commonly assumed that the quality and intensity of distinct olfactory stimuli is encoded by the combinatorial activation of different subsets of ORs and their cognate ORNs (Malnic et al., 1999).

\section{ANTENNAL LOBE}

Larval ORNs project into discreet glomeruli within the larval antennal lobe (LAL, Figure 2E). The projection pattern observed in the larval olfactory system is 1:1:1 with 21 ORNs (compared to millions in mice and humans) synapsing in 21 glomeruli innervated by $\sim 21$ second-order olfactory projection neurons (PNs) (Ramaekers et al., 2005). Notably, larval ORNs arising from the left and right DOs project purely ipsilaterally into the LAL (Heimbeck et al., 1999; Fishilevich et al., 2005) such that there is no cross talk between the left and right LALs.

In adults, ORNs of a single class project into discreet glomeruli within the adult antennal lobe (AL, Figure 2F) despite the fact that the ensemble of ORNs expressing the same OR are relatively scattered on the surface of the antenna (Laissue et al., 1999). The projection pattern observed is roughly 26:1:3 with $\sim 1300$ ORNs converging in $\sim 50$ glomeruli innervated by $\sim 150$ second-order PNs (Stocker et al., 1990; Tanaka et al., 2004; Couto et al., 2005). In contrast with larvae, many adult fly ORNs send axon collaterals across the midline and innervate like glomeruli in both the ipsilateral and contralateral ALs with similar synaptic strength (Stocker et al., 1990; Kazama and Wilson, 2008). In honeybees, the ORN-toglomeruli convergence is even more spectacular. Each antenna of the bee hosts 60,000 ORNs (Esslen and Kaissling, 1976) projecting onto roughly 160 glomeruli (Arnold, Masson et al., 1985). The AL is innervated by 800 PNs (Hammer, 1997) resulting in a corresponding projection ratio of $375: 1: 5$. Interestingly, and in contrast with adult Drosophila, bee ORNs innervate only their ipsilateral AL (Pareto, 1972; Mobbs, 1982).

As described, individual odors typically activate one or several subsets of ORN classes and as a consequence, the distribution of activated glomeruli within the larval and adult AL varies according to the identity and intensity of the stimulus (Fiala et al., 2002; Ng et al., 2002; Wang et al., 2003; Silbering and Galizia, 2007). Interestingly, several ORN classes have been shown to mediate innate attractive or aversive behaviors suggesting that olfactory preferences are segregated at the level of the AL (Suh et al., 2004; Kurtovic et al., 2007; Schlief and Wilson, 2007; Datta et al., 2008; Semmelhack and Wang, 2009).

In principle, increasing the number of primary ORNs from the larval to the adult life stage should reduce the effects of background noise arising from stochastic ORN activation. Computational modeling of ORN convergence onto discreet glomeruli in the adult $\mathrm{AL}$ has revealed that pooling inputs from $n$ independent ORNs can result in a signal-to-noise ratio (SNR) improvement of $\downarrow_{n}$ in PNs (Figure 2G) (Pearce et al., 2001). Indeed, within a single glomerulus every adult ORN synapses onto every $\mathrm{PN}$ resulting in a considerable convergence of many primary ORNs onto a few PNs (Kazama and Wilson, 2009). Although the actual enhancement obtained from such pooling by PNs is not as strong as theoretically predicted, it is nevertheless evident that increasing the absolute number of ORN inputs innervating a single glomerulus enhances the reliability of the PN response, and thus that of the ORN-PN signal transformation (Bhandawat et al., 2007).

Consistent with this idea, glomerular volume within the adult $\mathrm{AL}$ is not uniform between glomeruli but varies according to the number of primary ORN inputs (Couto et al., 2005; Kazama and Wilson, 2008) suggesting that SNR optimization varies among specific olfactory channels. Notably, PNs innervating large glomeruli have larger dendritic arbors than those innervating smaller glomeruli and, consequently, are harder to depolarize (Kazama and Wilson, 2008). However, due to the higher number of presynaptic ORN release sites, an inherently high probability of vesicle release in ORNs and a mechanism for homeostatic "size matching", ORN-PN signal transformations are stronger in larger golmeruli (Kazama and Wilson, 2008). Thus glomerular size appears to be a reliable indicator for selective olfactory specialization. Accordingly, glomeruli innervated by ORNs involved in pheromone detection in male flies have a relatively large volume in the AL and may confer a heightened sensitivity to female pheromones and improve mate selection (Stockinger et al., 2005). Likewise, olfactory specialists like 
Drosophila sechellia, which oviposit only on Morinda fruit, show enlarged glomeruli associated with the detection of this substrate (Dekker et al., 2006).

Although increasing the number of ORNs expressing the same OR represents an effective way to enhance sensitivity to specific odors, such specialization may come at a cost. An overrepresentation of a specific ORN class is apparently associated with a loss of other ORNs, possibly due to spatial constraints within the antenna and head or through the selective abolition of competing or irrelevant olfactory inputs (Dekker et al., 2006). These observations illustrate the ongoing selective influences on inter-glomerular and inter-species olfactory specialization.

Several classes of GABA-ergic inhibitory (Figure $\mathbf{2 H}$, larvae and adults) (Wilson and Laurent, 2005; Asahina et al., 2009) and cholinergic excitatory (Figure 2I, adults) (Olsen et al., 2007; Shang et al., 2007) local interneurons (LNs) in the AL receive input from both ORN axon terminals and $\mathrm{PN}$ dendrites, and reciprocally influence their activity. Calcium imaging in larvae (Asahina et al., 2009) and PN recordings in adults (Wilson and Laurent, 2005) have revealed that LNs are a source of non-linearity and contribute actively to the modulation of ORN to PN transformations. Some LNs appear to innervate every glomerulus and may modulate activity across the entire AL (Ng et al., 2002; Silbering and Galizia, 2007). Other LNs connect only a subset of glomeruli and may coordinate specific olfactory inputs with similar functions (Wilson and Laurent, 2005; Silbering and Galizia, 2007). Functional analyses have demonstrated that LNs can inhibit $\mathrm{PN}$ responses downstream of strong ORN input (Figure $\mathbf{2} \mathbf{H}$, inset) (Olsen and Wilson, 2008) or enhance $\mathrm{PN}$ responses downstream of weak ORN input (Figure 2I, inset) (Bhandawat et al., 2007). Also, LNs can activate PNs beyond the odor specificity of their cognate ORN inputs by distributing activity across the AL (Wilson et al., 2004). Combined, these mechanisms serve to modulate the overall gain and specificity of ORN-PN signal transformations in the AL (Olsen and Wilson, 2008).

In a behavioral context, the sequential recruitment of ORs with different affinities apparently expands the operating range of the fly olfactory system (Kreher et al., 2008; Asahina et al., 2009). For instance, wild type larvae display stable attraction to ethyl butyrate (ETB), a fruity odor emitted by pineapple. This behavior is mediated by three receptors (Or35a, Or42a and Or42b) which respond to ETB across concentrations covering three orders of magnitude (Asahina et al., 2009). As illustrated in Figure 2J, attraction to a given odor across a wide concentration range (Figure 2J, red) may be facilitated by several classes of ORs, each conferring behavioral sensitivity to a narrow concentration range (Figure 2J, blue, green, maroon). The lower limits of the response are determined by the OR affinity while the upper limits are fixed by the concentration at which the OR saturates and by the gain modulation generated by inhibitory LNs (Figure 2J, blue, green, maroon) (Olsen and Wilson, 2008). Mechanisms underlying concentration-invariant odor perception have been described elsewhere (Wilson and Mainen, 2006). Conceptually, the encoding of a continuum of stimulus intensities by an array of narrowly tuned receptors with overlapping receptive fields is similar to the tonotopic encoding of sound in auditory systems (Oxenham et al., 2004; Egorova and Ehret, 2008) and varying spectral sensitivities in visual systems
(Yankov et al., 1999; Gao et al., 2008). The extent to which odor perception in larval and adult flies is technically "concentration-invariant" remains to be determined. Associative learning has proven to be a powerful framework to address this question (Yarali et al., 2009).

\section{HIGHER-ORDER OLFACTORY PROCESSING CENTERS}

Although olfactory behaviors have been utilized to dissect peripheral circuitry (Fishilevich et al., 2005; Schlief and Wilson, 2007; Kreher et al., 2008; Semmelhack and Wang, 2009) very little is known about the precise sensory-motor transformations underlying these behaviors. In larvae, second-order cholinergic PNs project to higher brain regions known as the mushroom body (MB) and the lateral horn (LH) (Python and Stocker, 2002). Likewise, in adults some PNs project collaterals to both the MB and the LH of the protocerebrum, whereas others directly innervate the LH (Marin et al., 2002). These projections are highly stereotyped in the MBs and LHs and appear to maintain some of the odotopic segregation established in the both the larval and adult AL (Masuda-Nakagawa et al., 2005, 2009; Jefferis et al., 2007).

Electrophysiological studies in adult Drosophila have revealed that adult MB Kenyon cells (KCs) receive input from $\sim 10 \mathrm{PNs}$ (Turner et al., 2008) and at most, a single PN from the same glomerulus (Kazama and Wilson, 2009). This implies that convergence on KCs from specific olfactory channels is low. Rather, KCs receive sparse input from many different glomeruli. Although odor-evoked oscillations have been observed in KCs (Tanaka et al., 2009) and many extrinsic neurons from the MB have been identified (Tanaka et al., 2008), their precise influence on circuits controlling behavior is unknown. Alternatively, PNs from the same glomerulus converge in overlapping and distinct regions of the LH (Jefferis et al., 2007) suggesting that pooling by LH output neurons may further enhance the SNR of olfactory signals beyond that accomplished in the AL (Kazama and Wilson, 2009). While the $\mathrm{MB}$ has been implicated as a site for olfactory learning and memory and, in turn, experience-dependent modulation of olfactory behavior (Heisenberg et al., 1985; Ito et al., 1998; Zars et al., 2000; Heimbeck et al., 2001; Gerber et al., 2004; Krashes et al., 2007), the LH appears to be a site for innate, experience-independent olfactory behavior and multisensory integration (Heimbeck et al., 2001; Tanaka et al., 2004; Duistermars and Frye, 2010). Further knowledge about higher-order circuit control of olfactory behavior remains incomplete.

\section{ENHANCED OLFACTORY PERCEPTION WITH MULTIPLE SENSORS}

Sound localization involves interaural time differences and depth perception requires stereo vision. Considering the fact that the larval and adult olfactory systems comprise two bilaterally symmetric olfactory organs (Figures 2A,B,E,F), one can only wonder whether there is an advantage in processing odor cues with spatially distributed sensors. In larvae and adults, doubling the amount of input by adding a second, spatially separated sensor, might reduce correlated background noise between the two sensors and further improve the SNR compared to a single sensor alone (Bhandawat et al., 2007; Louis et al., 2008a). In addition, enhanced olfactory 
perception might arise from the measurement of spatial gradients through bilateral olfactory input. This computational advantage could be key for spatial orientation tasks where, for instance, flies need to rapidly navigate toward a food source or potential mate. It is conceivable that instantaneous odor samplings by two spatially segregated sensors permits flies to instantly approximate the direction of an attractive (or repulsive) chemical gradient, and thus allow for an appropriate locomotor response towards (or away from) the signal.

\section{DO LARVAEUSE COMPARISONS BETWEEN THE LEFT AND RIGHT OLFACTORY INPUTS?}

To navigate in odor gradients, it has been hypothesized that the larval brain takes advantage of concentration differences between the left and right DOs (Bala et al., 1998; Cobb, 1999). To experimentally assess how larvae integrate olfactory signals, controlled odorant gradients were created in closed arenas comprising multiple odor sources (Louis et al., 2008b). The geometry and stability of the gradients were measured using a novel method based on Fourier Transform Infrared (FT-IT) spectroscopy (Louis et al., 2008) (Figure 3A). By establishing a correlation between the stimulus conditions and behavioral responses, larvae were shown to chemotax according to a direct orientation mechanism where the direction of motion is aligned with the vector field of the odor gradient (Louis et al., 2008a). This strategy is in stark contrast with the biased random walks implemented by bacteria and nematodes (see "Modes of chemotaxis").

By combining genetic tools available to manipulate the peripheral olfactory system of the fly, larvae with a single functional class of ORNs were generated by selectively rescuing OR83b expression with $O R-G a l 4$ drivers in an OR83b null background (Fishilevich et al., 2005). These experiments demonstrate that larvae carrying a single functional class of ORNs are capable of robust chemotaxis to a reduced subset of odors. To experimentally test the relevance of stereo odor detection, a genetic strategy was devised to stochastically rescue OR83b expression in an individual ORN on the left, right, or both side(s) of the head (Figure 3B). Using these transgenic larvae, the chemotactic performances of individuals with unilateral and bilateral olfactory inputs were measured in various conditions (Louis et al., 2008a). These experiments revealed that unilateral function is sufficient to mediate significant chemotaxis (Figures 3B,C). This striking finding rules out orientation models based purely on left-right comparisons (osmotropotaxis) even though it does not exclude its contribution.
A

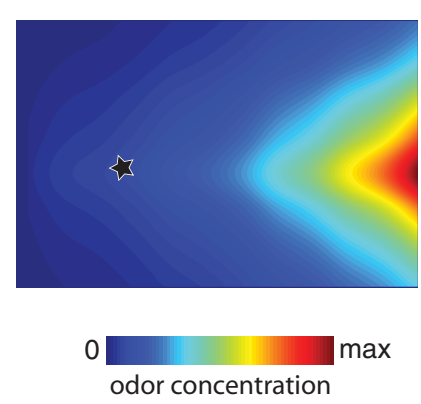

B
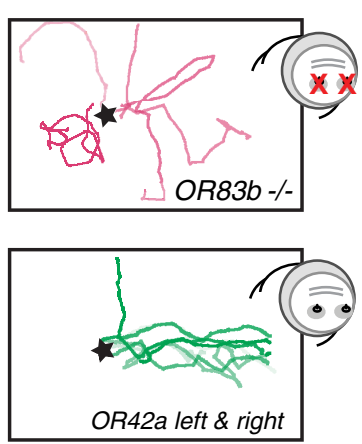
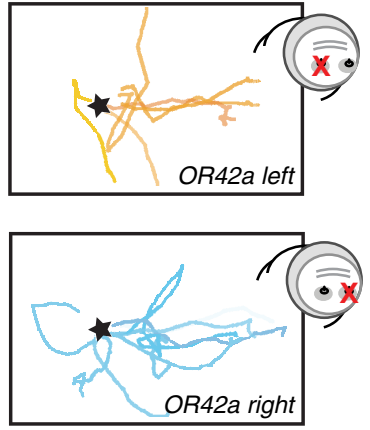

C

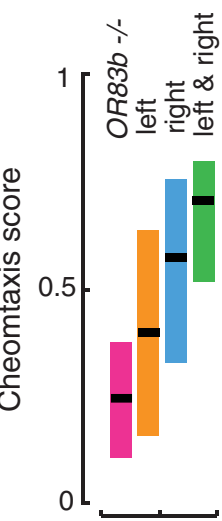

D Stimulus

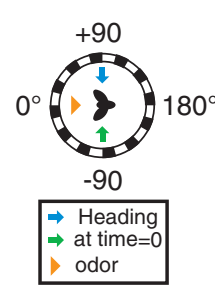

E Control

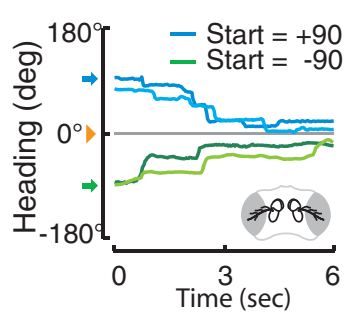

F Left antenna occluded (LO) $180^{\circ}$

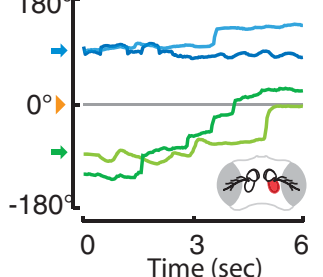

G

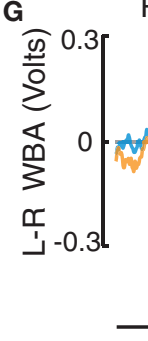

Rigid tether flight arena

Turn direction: right
FIGURE 3 | Behavioral performances of Drosophila with unilateral olfactory function. (A-C) Drosophila larvae do not require bilateral sensory input for chemotaxis. (A) Topographic reconstruction of a controlled exponential odor gradient based on infrared spectroscopy (for details see Louis et al., 2008a). The star represents the location in the arena where the larva is introduced. (B) Representative experimental trajectories of OR83b null mutants (red) and larvae with olfactory input rescued bilaterally (green) or unilaterally in a single functional OR42a-expressing ORN (left, orange; right, blue). (C) Quantification of chemotactic performance with a score measuring the overall alignment of the trajectory with the gradient (data of panels A-C are adapted from Louis et al., 2008a). (D-G) Adult flies use bilateral spatial comparisons of antennal inputs for spatial orientation in flight. (D) In a free-yaw magnetic tether flight arena, flies were positioned $90^{\circ}$ to the right (+, blue arrows) or left (-, green arrows) of a spatially discreet odor plume (orange triangle). (E) The resulting flight trajectories, where color is used to discriminate between individuals, reveal that flies actively turn toward the odor plume. (F) Occluding the left 3rd antennal segment with UV activated glue (red) impairs olfactory orientation to the left (blue), but not to the right (green). (G) When exposed to a fixed head-on odor plume in a rigid tether flight arena, unilaterally occluded flies modulate left and right wing beat amplitude (WBA) in attempt to steer toward the intact antenna (data of panes $\mathbf{D}-\mathbf{G}$ are adapted from Duistermars et al., 2009). 
Since larvae carrying a single functional ORN on one side of the head are not dramatically impaired in their chemotactic ability, do stereo inputs have any behavioral relevance? As shown in Figure 3C, bilateral olfactory input enhances the precision of the orientation response. Three hypotheses have been proposed to explain this observation: (1) doubling the number of ORNs decreases the sensory threshold of the system; (2) the larval brain is able to detect small concentration differences between left and right sides; (3) pooling the signal from two ORNs increases the SNR and thus the reliability of sensory-motor transformations. Point (1) was ruled out experimentally as unilateral larvae were tested at concentrations higher than the sensory threshold (Louis et al., 2008a). Both points (2) and (3) stand as valid options. However, in spite of the attractive parallelism that point (2) creates with the visual and auditory systems, this idea is not supported by a simple order of magnitude estimate.

Given that the DOs have a typical diameter $a=5 \mu \mathrm{m}$ and are separated approximately by a distance $d=150 \mu \mathrm{m}$ (Cobb, 1999), we can estimate the number of molecules in each DO and compare the difference between the left and right sensors for realistic linear gradients. When exposed to an odor concentration of $C=50 \mu \mathrm{M}$, the average number of molecules in one DO is on the order of $\langle\mathrm{N}\rangle \sim C \times a^{3} \approx 4 \times 10^{6}$. Assuming individual odorant molecules randomly enter into the DO, the measurement of $\langle\mathrm{N}\rangle$ has an associated noise of $\delta N \sim \sqrt{ }\langle\mathrm{N}\rangle \approx 2 \times 10^{3}$ molecules. For simplicity, we neglect convection or turbulences in the medium. When considering a gradient with a slope $s=3 \mu \mathrm{Mcm}^{-1}$ (Louis et al., 2008a), the inter-nasal concentration difference $\Delta C=s \times d$ is $45 \mathrm{nM}$ when the larval head lies perpendicular to the gradient. This corresponds to a mean difference in the number of molecules between the left and the right sensory organs of $\Delta N=\Delta C \times a^{3} \approx 3 \times 10^{3}$ molecules. Since this mean difference is comparatively similar to the associated noise, fluctuations in the detection of odorant molecules set a physical limit on detection accuracy (Berg and Purcell, 1977; Bialek and Setayeshgar, 2005). Therefore, provided an exquisite sensitiveness of the larval olfactory system, the above estimates indicate that it is unlikely that larvae perform reliable measurements of such concentration differences through direct instantaneous bilateral comparisons.

Since larval ORNs do not project contra-laterally in the LAL, it is conceivable that an elementary form of SNR improvement takes place in the larval olfactory system where merging the input from two physically separated ORNs might increase the detection performance by a factor of $\sqrt{ } 2$. Although this latter hypothesis seems likely, we still do not know where the information arising from the left and right pathways is merged and transformed into asymmetrical motor output.

Finally, as larval chemotaxis does not require stereo sampling (Figure 3B) and results from a direct orientation process, the following question naturally arises: how are larvae with unilateral olfactory function capable of sensing whether an odor gradient is on the left or right? High-resolution behavioral analyses suggest that olfactory conditions are sampled at different points in space: by translating their ORNs in space, larvae might be able to estimate the direction of highest concentration change in their vicinity (Louis et al., 2008a). Presumably, this active sampling is achieved during bouts of lateral head movements with about one side-to-side scan occurring each second (A. Gomez-Marin, G. Stephens and M. Louis, unpublished data). Head scans result in a reiterative and sequential alignment of the direction of motion with that of the odor gradient. Whether concentrations measured at different points are integrated peripherally or centrally remains unclear.

\section{DO ADULTSUSE COMPARISONS BETWEEN LEFT AND RIGHT OLFACTORY INPUTS?}

Behavioral evidence suggests that adult flies are able to perceive and respond to stereo olfactory cues. Walking adult flies have been observed orienting in relation to an olfactory gradient delivered across the two antennae (Borst and Heisenberg, 1982). Likewise, flies encountering an odor plume in free flight are able to redirect their flight heading within $250 \mathrm{~ms}$ of plume contact (Budick and Dickinson, 2006), suggesting that spatial olfactory comparisons are rapid and robust.

Recently, observations of flight in a free-yaw magnetic tether in which animals are fixed to a metal pin, suspended within a magnetic field, and free to rotate horizontally (Duistermars and Frye, 2008), have provided new insights into this phenomenon. Flies positioned $90^{\circ}$ to the right or left of an attractive apple cider vinegar odor plume (Figure 3D) execute turning maneuvers directed toward the odor stimulus (Figure 3E) (Duistermars et al., 2009). Occluding the left 3rd antennal segment with glue, and thus removing olfactory input to the left antennal ORNs, impairs olfactory orientation to the left (occluded side) but not to the right (non-occluded side) and visa versa (Figure 3F). Similarly, in a rigid tether flight simulator equipped with a head-on plume (method detailed in Frye and Dickinson, 2004), unilaterally occluded flies display attempted turns, or asymmetrical modulations in wing beat amplitude, toward the non-occluded antenna (Figure 3G). These directed maneuvers are activated only in the presence of odor, mitigating any potential non-specific effects of the glue, and indicate that antennal ORNs are the primary inputs for detecting asymmetrical odor gradients in flight.

It appears that both walking and flying adults can use spatial olfactory cues for orientation into an odor gradient. However most ORNs, including those thought to mediate behavioral responses to apple cider vinegar (Semmelhack and Wang, 2009), project bilaterally to both antennal lobes (Stocker et al., 1990). Also, recordings from PNs downstream of bilateral ORNs in response to unilateral stimulation of the antennal nerve reveal equal synaptic strengths in ipsilateral and contralateral glomeruli (Kazama and Wilson, 2008) and a contralateral delay of $\sim 0.3 \mathrm{~ms}$ (Kazama and Wilson, 2009). With these apparent spatial and temporal constraints, how do flies compare olfactory information between the two antennae? Analysis of spontaneous and odor induced activity in homotypic ipsilalateral and contralateral PNs in response to bilateral olfactory stimulation reveal a subtle possibility (Kazama and Wilson, 2009). Correlation in both pairs is strong, but notably weaker in contralateral $\mathrm{PN}$ pairs, a difference which could be greater in response to unilateral or asymmetrical odor stimulation and further amplified in subsequent stages of signal processing. As another interesting point, deoxyglucose labeling has demonstrated that AL activation in response to unilateral antennal stimulation with attractive odor cues is restricted 
to ipsilateral glomeruli (Rodrigues, 1988). In contrast, unilateral stimulation with repulsive odor cues of similar concentration activates both ipsilateral and contralateral glomeruli suggesting that spatial comparisons may be a unique feature of attractive olfactory behaviors. Although this result may be confounded by differing stimulus detection thresholds, no mechanism has yet been described whereby ipsilateral and contralateral glomeruli are serially recruited in response to increased odor concentrations. In total, the precise mechanisms for extracting spatial information from bilateral ORNs are still undetermined.

\section{FURTHER CONSIDERATIONS FOR DROSOPHILA OLFACTORY BEHAVIORS}

When analyzing peripheral olfactory circuits by way of electrophysiology and anatomy, it is possible to draw spurious conclusions regarding their contribution to behavior. It is paradoxical that freely crawling larvae do not require bilateral comparisons to track a spatial gradient despite having, like adult bees, the fully unilateral sensory projections that would enable it (Louis et al., 2008). In contrast, despite the bilateral projections of most ORNs in adults, flies use bilateral comparisons to track odor plumes in flight (Duistermars et al., 2009). Thus, conclusions drawn from circuit-function analyses should be considered in the context of stereotypical behavioral tasks.

One characteristic of olfaction in adult Drosophila is the apparent separation of attractive and aversive olfactory pathways. Specific ORNs and in turn, their cognate PNs appear to be odotopically segregated in deep brain regions (Jefferis et al., 2007) and innately hard-wired to trigger attractive or aversive behaviors (see "Antennal lobe"). Olfactory information encoded by such labeled lines could be combined hierarchically to modulate odor-evoked behaviors. For instance, walking D. melanogaster females display a reduced behavioral attraction to apple cider vinegar in the presence of $\mathrm{CO} 2$ (Faucher et al., 2006), while aversion to CO2 is reduced in the presence of specific food odors (Turner and Ray, 2009). However, these results, combined with the strict anatomical segregation of preference, may also suggest that the two modes of behavior are processed by competing, parallel pathways. Thus, while inhibition of attractive olfactory channels may conceptually produce the logical inverse of behavioral attraction (Kreher et al., 2008), activation of aversive channels may yield qualitatively different behavioral outcomes. As such, the behavioral algorithms for attraction may not be readily applicable to aversion.

It currently remains unclear how the electrophysiological properties of ORNs vary between active walking and flight. Active flight influences the velocity of air flow across the antennae and may in turn alter the functional "leakiness" of sensillar arrays, the time in which odorant molecules reside near the surface of olfactory sensilla, and the rate at which odorant molecules are encountered (Koehl, 2006). Interestingly, flying flies in a magnetic tether track an attractive odor plume with high accuracy despite the fact that the beating wings pass through and presumably perturb the plume (Figure 3E) (Duistermars et al., 2009). It would thus appear that the strong downwash induced by bilaterally flapping wings (Sane, 2006) may expand a fly's sampling space and spatially draw in or "focus" a discontinuous and asymmetrical natural plume (Vickers, 2000). Also, the beating wings deliver odiferous air onto the antennae in pulses at wing beat frequency (Loudon and Koehl,
2000). In moths, ORN spike timing is entrained with pulsed odor plumes that approximate natural wing beat frequencies (WBF) up to $30 \mathrm{~Hz}$, enhancing perceptual sensitivity (Tripathy et al., 2010). In Drosophila, however, the high WBF (>200 Hz) may enhance flow locally across the antennae without inducing temporal fluctuations in the ORNs.

Curiously, flies tonically increase WBF in response to attractive apple cider vinegar or banana odor (Frye and Dickinson, 2004; Budick and Dickinson, 2006). This may be conceptually similar to wing fanning by silk worm moths (Loudon and Koehl, 2000) and may confer an odor-evoked enhancement of odor sensitivity in flight due to the scaling of downwash velocity with WBF (Sane, 2006). It has yet to be determined whether an odor mediated increase in WBF resembles a "sniff", reduces the "time to arrival", or is the result of other processes known to act on WBF (David, 1982; Duistermars and Frye, 2010). Finally, recent studies have revealed that the gain of visual processing circuits is acutely enhanced during flight (Maimon et al., 2010). The adult $\mathrm{AL}$ is subject to neuromodulation by serotonergic inputs from higher brain regions (Dacks et al., 2009), and it is therefore conceivable that flight evoked modulation of the AL may contribute to enhanced olfactory sensitivity.

\section{FROM CIRCUITS TO BEHAVIOR: SPECULATIONS ON VIRTUAL VEHICLES}

To illustrate the basic principles capturing the phenomenology observed in bacteria, worms, larval fly, and adult fly chemotactic behaviors, it is useful to consider an approach based on thought experiments by biocyberneticist Valentino Braitenberg (Braitenberg, 1984). Braitenberg proposed a framework where autonomous machines (vehicles) interact with their environment based on relatively simple stimulus-response rules. The rich repertoire of behaviors displayed by the wide variety of these vehicles led Braitenberg to conclude that sensory-motor mechanisms presumed to be overwhelmingly complex can instead arise from a combination of basic commands. Thus, using pure abstraction, we aim to identify simple algorithms permitting motile organisms to navigate toward an attractive odor source.

\section{BIOLOGICALLY INSPIRED VEHICLES}

We have designed three chemotactic vehicles qualitatively inspired by the sensory circuits and anatomical constraints of bacteria, larval and adult flies. Here, we describe these vehicles in accordance with some specific examples put forth by Braitenberg. Further, we propose a minimal set of orientation rules which qualitatively capture the main features of the experimentally observed behaviors (Figures 1 and 3). Considering our limited understanding of sensory-motor transformations in these animals, we will avoid explicit parallels between these vehicles and the actual biological systems.

Our type A vehicle stems from a simplified version of the chemotaxis algorithm of E. coli and C. elegans and is similar to Braitenberg's "Vehicle 1" (Figure 4A). This vehicle is equipped with a single sensor directly controlling a single motor such that more sensor activation results in more motor activation. It also modulates the frequency of random turns in a way that is inversely proportional to the concentration. It can be said to abide by the following simple rules: If the concentration is low turn frequently. 
A E.coli; C. elegans
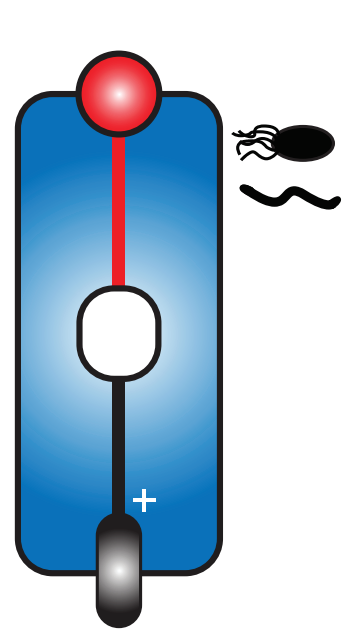

B larval Drosophila

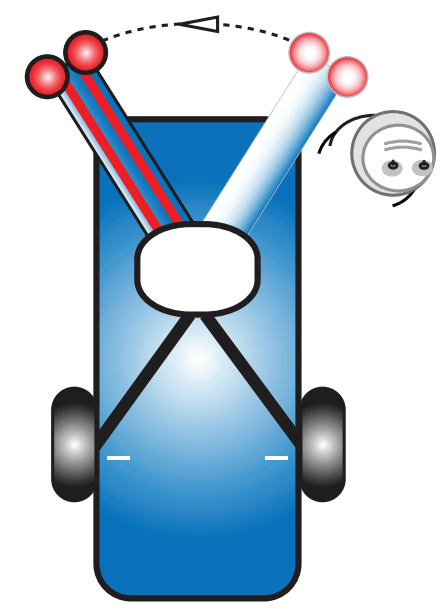

FIGURE 4 | Design of three main classes of Braitenberg-like vehicles inspired by real biological systems. (A) Our type A vehicle is akin to E. coliand C. elegans and corresponds to the original Braitenberg's "Vehicle 1" (Braitenberg, 1984, pp. 3-5). This vehicle has a single fixed sensor which directly activates a single motor. (B) Our type B vehicle, analogous to Drosophila larvae, is similar to Braitenberg's "Vehicle 3a" (Braitenberg, 1984, pp. 10-12). It has two sensors in close proximity at the tip of a laterally swinging head and sensor activation
Conversely, if the concentration is high suppress random turns and go straight. For this vehicle, turning rate and velocity are a function of stimulus intensity.

Our type B vehicle, akin to Drosophila larvae and similar to Braitenberg's "Vehicle 3a", is more complex in its processing and posture control. As the vehicle moves forward, its mobile head swings laterally at a constant frequency to explore the environment (Figure 4B). Two identical and independent sensors are positioned at the tip of the head and information arising from both sensors is combined within the processor. Two independent motors are driven by the processor such that more sensor activation results in less motor activation. This vehicle abides by the following rule: When the head is engaged to the side, inhibit the ipsilateral motor at a level proportional to the sensory input. As the vehicle moves forward, motor commands are reiteratively issued while proprioceptive sensors signaling the extreme lateral positions of the head are integrated with local stimulus intensities from the sensors to unilaterally affect motor output.

Our type $C$ vehicle, inspired by flying Drosophila adults and similar to Braitenberg's "Vehicle 2b", is equipped with two spatially segregated sensors which independently and directly excite their contralateral motors such that more sensor activation results in more motor activation (Figure 4C). Similar to velocity control in flight, this vehicle has a basal level of motor activation which is modulated by sensory input. This vehicle abides by the following rules: If the difference in concentration across the two sensors is zero, increase the activation of both motors at a level proportional to the sensory input. Conversely, if a concentration difference is measured across the two sensors, activate more strongly the motor opposite the side of highest odor concentration. This asymmetrical motor activation induces a turn towards the highest concentrations of odor.
C adult Drosophila

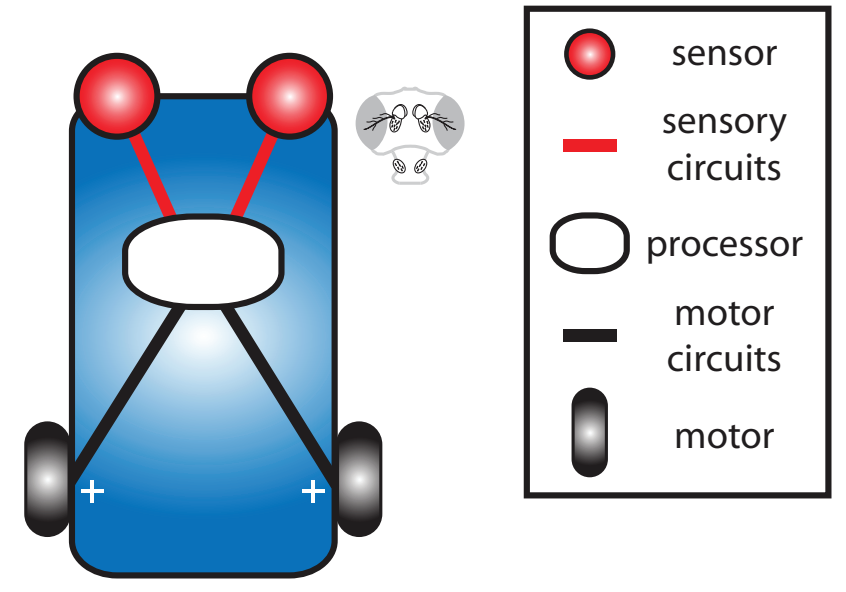

influences motor activity when the head is engaged to one side. (C) Our type C vehicle is inspired by flying adult Drosophila and corresponds to Braitenberg's "Vehicle 2b" (Braitenberg, 1984, p. 7). It is able to detect concentration gradients via two spatially distributed sensors which directly activate contralateral motors. Processors can contain sites for integration of proprioceptive feedback (type B) and include more realistic specific stimulus thresholds, filters, non-linear dependencies on stimulus intensity, and sites for elementary memory.

\section{VEHICLES IN MOTION}

By virtue of our vehicles' idiosyncratic characteristics, the presence of an odor gradient induces motion according to our described set of sensory-motor rules. From a geometrical point of view, it is not possible to determine the direction of a vector from a single scalar measurement. In other words, placing one sensor at a particular point in space and measuring the concentration at this precise location is not enough to obtain a rough estimate of the direction towards the odor source. Therefore, in the absence of comparisons between multiple sensors scattered along the body of an animal, active spatial sampling is a sine qua non for direct orientation. Together, the "behavior" of our vehicle types A, B and C illustrate three fundamentally different orientation strategies.

A type A vehicle exhibits motion which results from an alternation between two modes of behavior: straight displacements and turns randomizing its orientation. In this "run and turn" paradigm, the probability of entering either mode of action is directly related to the local stimulus intensity. As this vehicle cannot compute the direction of odor gradients, the source is approached as a result of a net accumulation of indirect reorientations (kinesis) (Figure 5, type A). However, as this vehicle's motor is activated by high stimulus concentrations it would be expected to overshoot the odor source, and in this sense can be considered, as Braitenberg termed this phenomenon, "alive" (Braitenberg, 1984). The remarkable ability of E. coli to accumulate at the source of attractive gradients (Berg, 2004) points out the limit of our analogy with the type A vehicle. In reality, the adaptability of the chemosensory transduction pathway confers a high precision and robustness to the "run and turn" paradigm implemented by bacteria (Berg and Brown, 1972). 


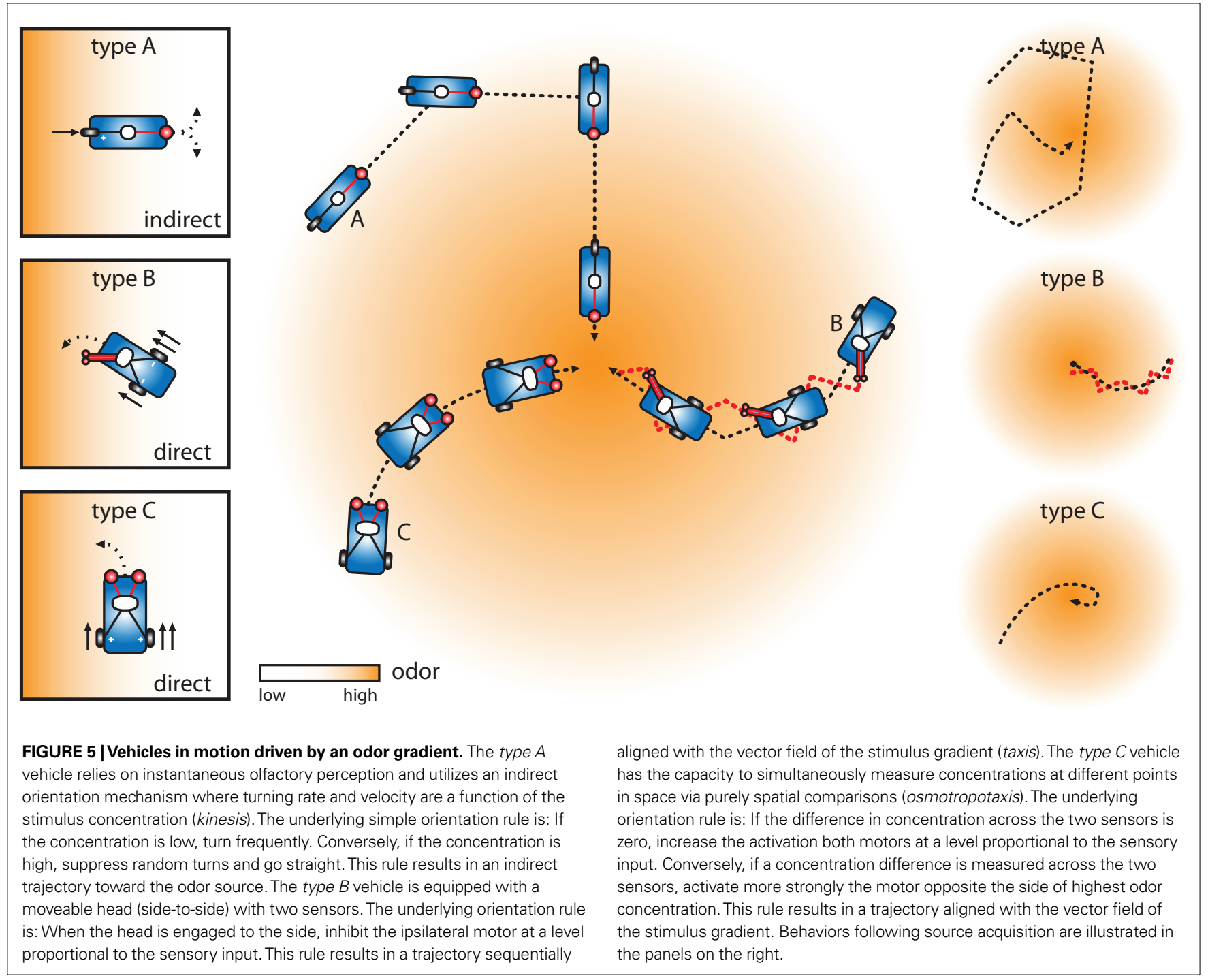

A type $B$ vehicle is unable to compute spatial asymmetries in the stimulus distribution instantaneously. Instead, it integrates signals from the sensors and proprioceptive inputs to unilaterally influence motor activity. To estimate the direction of the gradient, the vehicle combines information about the signal strength and the orientation of the head (Figure 5, type B). As a result of this sequential, unilateral inhibition of motor output, the motion kinetics of this vehicle is directly modulated by the geometry of the gradient (taxis). Since sensory input inhibits motor output, type B stays in motion in the absence of a stimulus, a feature reminiscent of foraging behavior in larvae. Furthermore, this vehicle is expected to slow down, or stop, when concentrations are high. In analogy with the compelling attraction of larvae to many odors, the type B vehicle "loves" the odor source (Braitenberg, 1984).

A type $C$ vehicle is capable of instantaneously evaluating stereo information via a pair of spatially distributed sensors. Due to the direct contralateral excitation of motor output, a type $\mathrm{C}$ vehicle accurately follows the field of the gradient. This navigation process makes direct use of the spatial gradient (osmotropotaxis) and is arguably the most economic path to reach the odor source (Figure 5, type C). As odor cues excite motor output, this vehicle is expected to accelerate past an attractive odor source of high concentration, and in this sense, is reminiscent of flying flies encircling an odor source. This behavior can be considered "aggressive" (Braitenberg, 1984).

\section{FURTHER CONSIDERATIONS ON BRAITENBERG'S VEHICLES}

As Braitenberg suggests, it is relatively easy to design machines performing complex behaviors, whereas reverse-engineering the internal structure of a vehicle from its behavior is more difficult. The biological correlate of this conclusion is that neuronal functions controlling simple behaviors cannot be unraveled without prior knowledge about the circuit structure. However, models and assumptions are a valuable experimental tool to examine experimental data and formulate predictions. Using abstract considerations, we can draw several conclusions on the general principles underlying olfactory perception and behavior.

For instance, the different orientation mechanisms featured by type A, B and C vehicles are not mutually exclusive. Rather, they can be combined and expanded in a modular fashion. In response to fluctuating stimuli, shallow gradients, damaged sensors or complex 
sensory environments, an organism may switch from one algorithm to another or use them simultaneously. As an example, and similar to the observed transition from osmotropotaxis to klinotaxis in bees (see "Evidence for the use of stereo olfaction in insects and vertebrates") (Martin, 1965), a type C vehicle performing effective bilateral comparisons could switch to active head movements, a type B strategy, if the distance between its sensors is reduced. Also, flying flies utilize a type C spatial strategy (Figure 3E) but behavioral studies suggest this operates in tandem with a bacteria-like type A strategy. In the absence of odor, flying flies exhibit spontaneous turning maneuvers closely approximating an optimal scale-free search strategy (Reynolds and Frye, 2007). Attractive odor cues suppress the overall frequency of turning maneuvers and facilitate forward flight (Budick and Dickinson, 2006; Duistermars and Frye, 2008) indicating that flying adults roughly follow the rules of type A and type $\mathrm{C}$ vehicles.

In larvae ascending an odor gradient, no experimental evidence indicates that the speed or turning frequency is modulated by the stimulus intensity (Louis et al., 2008a) ruling out the use of a type A-like behavior. Thus it would appear, although we cannot completely rule out the capacity for type $\mathrm{C}$ behavior (see "Do larvae use comparisons between the left and right olfactory inputs?"), larvae may be restricted to type B actions. While modular stacking and behavioral plasticity may increase the resilience of a vehicle, larvae demonstrate that it may not be required for successful odor localization.

The simple rules underlying the motion of Braitenberg vehicles are not limited to those we have described. Indeed, Braitenberg's strategies can be expanded to include thresholds for and nonlinear dependencies on stimulus intensities (Braitenberg, 1984). For example, vehicles can withhold action until a stimulus reaches a set threshold, a phenomenon similar to the thresholds set by Drosophila ORs and ORNs (see "Odorant receptors and first-order olfactory receptor neurons"). Furthermore, vehicle output may increase linearly with stimulus intensity but saturate and decline with increasingly intense stimuli, a concept similar to that observed behaviorally in flies as a result of gain modulation in the AL (see "Antennal lobe"). Together, these two simple considerations, combined with sensors containing receptors with multiple ORs of varying affinities, contain properties necessary to confer concentration-invariant odor perception to our vehicles. Finally, the capacity to learn, or have a memory, could significantly enhance the success of olfactory tracking. For instance, type A vehicles do not exactly replicate bacteria or worms as they lack the capacity for evaluating $\Delta C / \Delta t$. This capacity could be included as an elementary form of memory which stores measurements of odor concentration over time.

\section{CONCLUDING REMARKS}

Observations of natural animal behavior are a rich source of information for developing computational algorithms that perform sensory tasks. To understand olfactory orientation behavior, it is important to go beyond a reductionist approach treating components of the olfactory system in isolation. Guided by the principle that the properties of neural circuits are revealed in the behaviors they mediate, we stress that high-resolution tracking and quantitative analysis of olfactory behaviors offer a valuable entry point into the hidden sensory mechanisms and neural processes underlying chemotaxis (Pierce-Shimomura et al., 1999; Louis et al., 2008a; Stephens et al., 2008; Duistermars et al., 2009). Although the simple rules governing Braitenberg's vehicles help capture essential features underpinning odor localization strategies, efforts to model the actual structure-function relationships must consider several factors including external and internal noise, limitations of experimental analysis, internal motivational state, and more generally, the influence of multiple sensory modalities.

Given the sparse and turbulent nature of natural odor stimuli, the intrinsic noisiness of biological sensors, and the imperfect implementation of neural algorithms, models of odor localization strategies will need to include the effects of stochasticity in the searching algorithms (Vergassola et al., 2007). The remarkable capacity of larvae and adult flies to localize odors in their environments is a consequence of the evolution of sophisticated mechanisms successfully dealing with the effect of these different sources of noise. Such mechanisms, as described, can include OR optimization, more ORs, more ORNs, SNR optimization, multiple sensors, and spatially distributed sampling.

The stimulus-response characteristics of a fly under experimental conditions can be contingent upon several factors. The nature of an odor stimulus whether delivered as a steady gradient, a series of temporal pulses or as a turbulent plume should attempt to replicate natural stimuli ecologically relevant to life stage and mode of motility. Also, experimental paradigms which constrain the animal should consider critically, if any, sensory modalities are impaired. For instance, rigid tether flight experiments (Figure 3G) necessarily impair gyroscopic feedback from self-generated motion, but leave visual, olfactory, mechanosensory and propriceptive inputs intact. Furthermore, many intrinsic factors condition the perceptual salience of a sensory input. Such factors include experience-induced plasticity, starvation, circadian rhythms, gender, mating states, genetic background and phenotypic variations, all of which can significantly influence experimental results and their interpretation.

The modeling of more complex strategies employed by animals with sophisticated neural circuitry may be confounded by the dependence of information from multiple sensory modalities. For instance, larvae respond to a wide variety of sensory stimuli including light, heat, chemical tastants and possibly humidity and gravity (Lilly and Carlson, 1990; Rosenzweig et al., 2005; Gerber and Stocker, 2007). The algorithms described here for enhanced odor perception and behavior are likely to be relevant to other modalities such as phototaxis and thermotaxis. The influence of these modalities on one another is largely unknown. Additionally, flying flies are unable to locate the source of an odor in the absence of rich visual feedback (Frye et al., 2003; Duistermars and Frye, 2008) and the capacity for spatial comparisons requires both visual feedback (Duistermars and Frye, 2010) and primary input from the mechanosensory Johnston's organ (Duistermars et al., 2009). Finally, as flies are highly multi-modal it is our hope that further experimentation on both ends of sensory-motor transformations will enable the deconstruction of the apparent complexity of fly behavior into simplified Braitenberg-like algorithms, which can simplify the current state-of-the-art while simultaneously generating testable hypotheses about the unknown. 


\section{ACKNOWLEDGMENTS}

Alex Gomez-Marin is supported by the Juan de la Cierva program from the Spanish Ministry of Science and Innovation. Alex GomezMarin is grateful to the Boehringer-Ingelheim Foundation for a Travel Grant to attend the "Neurobiology of Drosophila" course at the Cold Spring Harbor Laboratory. Matthieu Louis acknowledges funding from the Spanish Ministry of Science and Innovation (MICINN, BFU2008-00362), and the EMBL-CRG Systems Biology Program. Mark A. Frye is a Howard Hughes Medical Institute Early Career Scientist and is funded by the National Science Foundation (IOS0718325). We are grateful to the comments of Troy Shirangi and two anonymous referees which allowed us to improve this manuscript.

\section{REFERENCES}

Abaffy, T., Malhotra, A., and Luetje, C. W. (2007). The molecular basis for ligand specificity in a mouse olfactory receptor: a network of functionally important residues. J. Biol. Chem. 282, 1216-1224.

Aceves-Pina, E. O., and Quinn, W. G. (1979). Learning in normal and mutant Drosophila larvae. Science 206, 93-96.

Adler, J. (1969). Chemoreceptors in bacteria. Science 166, 1588-1597.

Afshar, M., Hubbard, R. E., and Demaille, J. (1998). Towards structural models of molecular recognition in olfactory receptors. Biochimie 80, 129-135.

Arnold, G., Masson, C., and Budharugsa, S. (1985). Comparative study of the antennal lobes and their afferent pathway in the worker bee and the drone (Apis mellifera). Cell Tissue Res. 242, 593-605.

Asahina, K., Louis, M., Piccinotti, S., and Vosshall, L. B. (2009). A circuit supporting concentration-invariant odor perception in Drosophila. J. Biol. 8, 9.

Asahina, K., Pavlenkovich, V., and Vosshall, L. B. (2008). The survival advantage of olfaction in a competitive environment. Curr. Biol. 18, 1153-1155.

Bala, A. D. S., Panchal, P., and Siddiqi, O. (1998). Osmotropotaxis in larvae of Drosophila melanogaster. Curr. Sci. 75, 48-51.

Beltramí, M., Medina-Muñoz, M., Arce, D., and Godoy-Herrera, R. (2010). Drosophila pupation behavior in the wild. Evol. Ecol. 24, 347-358.

Benton, R., Sachse, S., Michnick, S.W., and Vosshall, L. B. (2006). Atypical membrane topology and heteromeric function of Drosophila odorant receptors in vivo. PLoS Biol. 4, e20. doi:10.1371/ journal.pbio.0040020.

Benton, R., Vannice, K. S., Gomez-Diaz, C., and Vosshall, L. B. (2009). Variant ionotropic glutamate receptors as chemosensory receptors in Drosophila. Cell 136, 149-162.

Berg, H. C. (2004). E. coli in Motion. New York: Springer.

Berg, H. C., and Brown, D. A. (1972). Chemotaxis in Escherichia coli analysed by three-dimensional tracking. Nature 239, 500-504.

Berg, H. C., and Purcell, E. M. (1977). Physics of chemoreception. Biophys. J. 20, 193-219.
Bhandawat, V., Olsen, S. R., Gouwens, N. W., Schlief, M. L., and Wilson, R. I. (2007). Sensory processing in the Drosophila antennal lobe increases reliability and separability of ensemble odor representations. Nat. Neurosci. 10, 1474-1482.

Bhandawat, V., Reisert, J., Yau, K. W. (2005). Elementary response of olfactory receptor neurons to odorants. Science 308, 1931-1934.

Bialek, W., and Setayeshgar, S. (2005). Physical limits to biochemical signaling. Proc. Natl. Acad. Sci. U.S.A. 102, 10040-10045.

Borst, A., and Heisenberg, M. (1982). Osmotropotaxis in Drosophila melanogaster. J. Comp. Physiol. A 147, 479-484.

Braitenberg, V. (1984). Vehicles: Experiments in Synthetic Psycology. Cambridge, MA: MIT Press.

Buck, L., and Axel, R. (1991). A novel multigene family may encode odorant receptors: a molecular basis for odor recognition. Cell 65, 175-187.

Budick, S.A., and Dickinson, M.H. (2006). Free-flight responses of Drosophila melanogaster to attractive odors. J. Exp. Biol. 209(Pt 15), 3001-3017.

Cho, W., Heberlein, U., and Wolf, F. W. (2004). Habituation of an odorantinduced startle response in Drosophila. Genes Brain Behav. 3, 127-137.

Cobb, M. (1999). What and how do maggots smell? Biol. Rev. 74, 425-459.

Cobb, M., Bruneau, S., and Jallon, J. M. (1992). Genetic and developmental factors in the olfactory response of Drosophila melanogaster larvae to alcohols. Proc. R. Soc. Lond., B, Biol. Sci. 248, 103-109.

Couto, A., Alenius, M., and Dickson, B. J. (2005). Molecular, anatomical, and functional organization of the Drosophila olfactory system. Curr. Biol. 15, 1535-1547.

Dacks, A. M., Green, D. S., Root, C. M., Nighorn, A. J., and Wang, J.W. (2009). Serotonin modulates olfactory processing in the antennal lobe of Drosophila. J. Neurogenet. 23, 366-377.

Datta, S. R., Vasconcelos, M. L., Ruta, V., Luo, S., Wong, A., Demir, E., Flores, J., Balonze, K., Dickson, B. J., and Axel, R. (2008). The Drosophila pheromone cVA activates a sexually dimorphic neural circuit. Nature 452, 473-477.
David, C. T. (1982). Compensation for height in the control of groundspeed by Drosophila in a new 'barber's pole' wind tunnel. J. Comp. Physiol. A Neuroethol. Sens. Neural. Behav. Physiol. 147, 485-493.

Dekker, T., Ibba, I., Siju, K. P., Stensmyr M. C., and Hansson, B. S. (2006) Olfactory shifts parallel superspecialism for toxic fruit in Drosophila melanogaster sibling, D. sechellia. Curr. Biol. $16,101-109$.

Duistermars, B. J., Chow, D. M., and Frye, M. A. (2009). Flies require bilateral sensory input to track odor gradients in flight. Curr. Biol. 19, 1301-1307.

Duistermars, B. J., and Frye, M. A. (2008). Crossmodal visual input for odor tracking during fly flight. Curr. Biol. 18, 270-275.

Duistermars, B. J., and Frye, M. A. (2010). Multisensory integration for odor tracking by flying Drosophila. Commun. Integr. Biol. 3, 1-4.

Egorova, M., and Ehret, G. (2008) Tonotopy and inhibition in the midbrain inferior colliculus shape spectral resolution of sounds in neural critical bands. Eur. J. Neurosci. 28, 675-692.

Ejima, A., Smith, B. P., Lucas, C., van der Goes van Naters, W., Miller, C. J., Carlson, J. R., Levine, J. D., and Griffith, L. C. (2007). Generalization of courtship learning in Drosophila is mediated by cis-vaccenyl acetate. Curr. Biol. 17, 599-605.

Esslen, J., and Kaissling, K.-E. (1976).Zahl und Verteilung antennaler Sensillen bei der Honigbiene (Apis mellifera L.), Zoomorphology 83, 227-251.

Faucher, C., Forstreuter, M., Hilker, M., and de Bruyne, M. (2006). Behavioral responses of Drosophila to biogenic levels of carbon dioxide depend on life-stage, sex and olfactory context. $J$. Exp. Biol. 209(Pt 14), 2739-2748.

Fiala, A., Spall, T., Diegelmann, S. Eisermann, B., Sachse, S., Devaud, J. M., Buchner, E., and Galizia, C. G. (2002). Genetically expressed cameleon in Drosophila melanogaster is used to visualize olfactory information in projection neurons. Curr. Biol. 12, 1877-1884.

Fishilevich, E., Domingos, A. I., Asahina, K., Naef, F., Vosshall, L. B., and Louis, M. (2005). Chemotaxis behavior mediated by single larval olfactory neurons in Drosophila. Curr. Biol. 15, 2086-2096.

Fishilevich, E., and Vosshall, L. B. (2005). Genetic and functional subdivision of the Drosophila antennal lobe. Curr. Biol. 15, 1548-1553.

Fraenkel, G. S., and Gunn, D. L. (1961). The Orientation of Animals, Kineses, Taxes and Compass Reactions. New York: Dover Publications.

Frye, M. A., and Dickinson, M. H. (2004). Motor output reflects the linear superposition of visual and olfactory inputs in Drosophila. J. Exp. Biol. 207(Pt 1), 123-131.

Frye, M.A., Tarsitano, M., and Dickinson, M. H. (2003). Odor localization requires visual feedback during free flight in Drosophila melanogaster. J. Exp. Biol. 206(Pt 5), 843-855.

Gao,S., Takemura, S.Y., Ting, C.Y.,Huang, S., Lu, Z., Luan, H., Rister, J., Thum, A. S., Yang, M., Hong, S. T., Wang, J. W., Odenwald, W. F., White, B. H. Meinertzhagen, I. A., and Lee, C. H. (2008). The neural substrate of spectral preference in Drosophila. Neuron $60,328-342$.

Gerber, B., and Stocker, R. F. (2007). The Drosophila larva as a model for studying chemosensation and chemosensory learning: a review. Chem. Senses $32,65-89$.

Gerber, B., Tanimoto, H., and Heisenberg, M. (2004). An engram found? Evaluating the evidence from fruit flies. Curr. Opin. Neurobiol. 14, 737-744.

Hallem, E. A., and Carlson, J. R. (2006). Coding of odors by a receptor repertoire. Cell 125, 143-160.

Hallem, E. A., Ho, M. G., and Carlson, J. R. (2004). The molecular basis of odor coding in the Drosophila antenna. Cell $117,965-979$.

Hammer, M. (1997). The neural basis of associative reward learning in honeybees. Trends Neurosci. 20, 245-252.

Hangartner, W. (1967). Spezifit it und Inaktiviernng des Spurpberomons yon Lasiusfuliginosus Latr. und Orientierung der Arbeiterinnen im Duftfeld. Z. oergl. Physiol. 57, 103-136.

Heimbeck, G., Bugnon, V., Gendre, N., Haberlin, C., and Stocker, R. F. (1999). Smell and taste perception in Drosophila melanogaster larva: toxin expression studies in chemosensory neurons. J. Neurosci. 19, 6599-6609. 
Heimbeck, G., Bugnon, V., Gendre, N., Keller, A. and Stocker, R. F. (2001). A central neural circuit for experienceindependent olfactory and courtship behavior in Drosophila melanogaster. Proc. Natl. Acad. Sci. U.S.A. 98, 15336-15341.

Heisenberg, M., Borst, A., Wagner, S., and Byers, D. (1985). Drosophila mushroom body mutants are deficient in olfactory learning. J. Neurogenet. 2, 1-30.

Hoare, D. J., McCrohan, C. R., and Cobb, M. (2008). Precise and fuzzy coding by olfactory sensory neurons. J. Neurosci. 28, 9710-9722.

Ito, K., Suzuki, K., Estes, P., Ramaswami, M., Yamamoto, D., and Strausfeld, N. J. (1998). The organization of extrinsic neurons and their implications in the functional roles of the mushroom bodies in Drosophila melanogaster Meigen. Learn. Mem. 5, 52-77.

Jefferis, G. S., Potter, C. J., Chan, A. M., Marin,E.C., Rohlfing, T., Maurer,C.R., Jr., and Luo, L. (2007). Comprehensive maps of Drosophila higher olfactory centers: spatially segregated fruit and pheromone representation. Cell 128, 1187-1203.

Joseph, R. M., Devineni, A. V., King, I. F., and Heberlein, U. (2009). Oviposition preference for and positional avoidance of acetic acid provide a model for competing behavioral drives in Drosophila.Proc. Natl. Acad. Sci. U.S.A. 106, 11352-11357.

Kazama, H., and Wilson, R. I. (2008). Homeostatic matching and nonlinear amplification at identified central synapses. Neuron 58, 401-413.

Kazama, H., and Wilson, R. I. (2009). Origins of correlated activity in an olfactory circuit. Nat. Neurosci. 12, 1136-1144.

Keller, A., Zhuang, H., Chi, Q., Vosshall, L. B., and Matsunami, H. (2007). Genetic variation in a human odorant receptor alters odour perception. Nature 449 , 468-472.

Koehl, M. A. (2006). The fluid mechanics of arthropod sniffing in turbulent odor plumes. Chem. Senses 31, 93-105.

Kopp, A., Barmina, O., Hamilton, A. M., Higgins, L., McIntyre, L. M., and Jones, C.D. (2008). Evolution of gene expression in the Drosophila olfactory system. Mol. Biol. Evol. 25, 1081-1092.

Krashes, M. J., Keene, A. C., Leung, B., Armstrong, J. D., and Waddell, S. (2007). Sequential use of mushroom body neuron subsets during Drosophila odor memory processing. Neuron 53, 103-115.

Kreher, S. A., Kwon, J. Y., and Carlson, J. R. (2005). The molecular basis of odor coding in the Drosophila larva. Neuron 46, 445-456.
Kreher, S. A., Mathew, D., Kim, J., and Carlson, J. R. (2008). Translation of sensory input into behavioral output via an olfactory system. Neuron 59 , 110-124.

Kurtovic, A., Widmer, A., and Dickson, B. J. (2007). A single class of olfactory neurons mediates behavioural responses to a Drosophila sex pheromone. Nature 446, 542-546.

Laissue, P. P., Reiter, C., Hiesinger, P. R., Halter, S., Fischbach, K. F., and Stocker, R. F. (1999). Three-dimensional reconstruction of the antennal lobe in Drosophila melanogaster. J. Comp. Neurol. 405, 543-552.

Laissue, P.P., and Vosshall, L. B. (2008). The olfactory sensory map in Drosophila. Adv. Exp. Med. Biol. 628, 102-114.

Larsson, M. C., Domingos, A. I., Jones, W. D., Chiappe, M. E., Amrein, H., and Vosshall, L. B. (2004). Or83b encodes a broadly expressed odorant receptor essential for Drosophila olfaction. Neuron 43, 703-714.

Lilly, M., and Carlson, J. (1990). smellblind: a gene required for Drosophila olfaction. Genetics 124, 293-302.

Loudon, C., and Koehl, M. A. (2000). Sniffing by a silkworm moth: wing fanning enhances air penetration through and pheromone interception by antennae. J. Exp. Biol. 203(Pt 19), 2977-2990.

Louis, M., Huber, T., Benton, R., Sakmar, T. P., and Vosshall, L. B. (2008a). Bilateral olfactory sensory input enhances chemotaxis behavior. Nat. Neurosci. 11, 187-199.

Louis, M., Piccinotti, S., and Vosshall, L. B. (2008b). High-resolution measurement of odor-driven behavior in Drosophila larvae. J. Vis. Exp. pii: 638.

Maimon, G., Straw, A. D., and Dickinson, M. H. (2010) Active flight increases the gain of visual motion processing in Drosophila. Nat. Neurosci. 13, 393-399.

Malnic, B., Hirono, J., Sato, T., and Buck, L. B. (1999). Combinatorial receptor codes for odors. Cell 96, 713-723.

Marin, E. C., Jefferis, G. S., Komiyama, T., Zhu, H., and Luo, L. (2002). Representation of the glomerular olfactory map in the Drosophila brain. Cell 109, 243-255.

Martin, H. (1965). Osmotropotaxis in the honey-bee. Nature 208, 59-63.

Masse, N. Y., Turner, G. C., and Jefferis, G. S. (2009). Olfactory information processing in Drosophila. Curr. Biol. 19, R700-R713.

Masuda-Nakagawa, L. M., Gendre, N., O'Kane, C. J., and Stocker, R. F. (2009). Localized olfactory representation in mushroom bodies of Drosophila larvae. Proc. Natl. Acad. Sci. U.S.A. 106, 10314-10319.
Masuda-Nakagawa, L. M., Tanaka, N. K., and O'Kane, C. J. (2005). Stereotypic and random patterns of connectivity in the larval mushroom body calyx of Drosophila.Proc. Natl. Acad. Sci. U.S.A. 102, 19027-19032.

Miyamoto, T., and Amrein, H. (2008). Suppression of male courtship by a Drosophila pheromone receptor. Nat. Neurosci. 11, 874-876.

Mobbs, P. G. (1982). The brain of the honeybee Apis mellifera. I. The connections and spatial organization of the mushroom bodies. Philos. Trans. $R$. Soc. Lond., B, Biol. Sci. 298, 309-354.

Monte, P., Woodard, C., Ayer, R., Lilly, M., Sun, H., and Carlson, J. (1989). Characterization of the larval olfactory response in Drosophila and its genetic basis. Behav. Genet. 19, 267-283.

Ng, M., Roorda, R. D., Lima, S. Q. Zemelman, B. V., Morcillo, P., and Miesenbock, G. (2002). Transmission of olfactory information between three populations of neurons in the antennal lobe of the fly. Neuron 36 463-474.

Olsen, S. R., Bhandawat, V., Bhandawat, V., and Wilson, R. I. (2007). Excitatory interactions between olfactory processing channels in the Drosophila antennal lobe. Neuron 54, 89-103.

Olsen, S. R., and Wilson, R. I. (2008) Lateral presynaptic inhibition mediates gain control in an olfactory circuit. Nature 452, 956-960.

Oppliger, F.Y., Guerin, P.M., and Vlimant, M. (2000). Neurophysiological and behavioural evidence for an olfactory function for the dorsal organ and a gustatory one for the terminal organ in Drosophila melanogaster larvae. J. Insect Physiol. 46, 135-144.

Oxenham, A. J., Bernstein, J. G., and Penagos, H. (2004). Correct tonotopic representation is necessary for complex pitch perception. Proc. Natl. Acad. Sci. U.S.A. 101, 1421-1425.

Pareto, A. (1972). Die zentrale Verteilung der Fühlerafferenz bei Arbeiterinnen der Honigbiene, Apis mellifera L. Cell Tissue Res. 131, 109-140.

Pearce, T. C., Verschure, P. F. M. J., White, J. and Kauer, J. S. (2001). "Robust stimulus encoding in olfactory processing: hyperacuity and efficient signal transmission," in Emergent Neural Computational Architectures Based on Neuroscience, Vol. 2036, eds S. Wermter, J. Austin, and D. J. Willshaw (Berlin: Springer), 461-479

Pellegrino, M., and Nakagawa, T. (2009). Smelling the difference: controversial ideas in insect olfaction. J. Exp. Biol. 212(Pt 13), 1973-1979.

Pierce-Shimomura, J. T., Morse, T.M., and Lockery, S. R. (1999). The fundamental role of pirouettes in Caenorhabditis elegans chemotaxis. J. Neurosci. 19 , 9557-9569.

Porter, J., Craven, B., Khan, R. M., Chang, S. J., Kang, I., Judkewitz, B., Volpe, J., Settles, G., and Sobel, N. (2007). Mechanisms of scent-tracking in humans. Nat. Neurosci. 10, 27-29.

Purcell, E. M. (1977). Life at low Reynolds number. Am. J. Phys. 45, 3-11.

Python, F., and Stocker, R. F. (2002). Adult-like complexity of the larval antennal lobe of D. melanogaster despite markedly low numbers of odorant receptor neurons. J. Comp. Neurol. 445, 374-387.

Rajan, R., Clement, J. P., and Bhalla, U. S. (2006). Rats smell in stereo. Science 311, 666-670.

Ramaekers, A., Magnenat, E., Marin, E. C., Gendre, N., Jefferis, G. S., Luo, L., and Stocker, R. F. (2005). Glomerular maps without cellular redundancy at successive levels of the Drosophila larval olfactory circuit. Curr. Biol. 15, 982-992.

Reynolds, A. M., and Frye, M. A. (2007). Free-flight odor tracking in Drosophila is consistent with an optimal intermittent scale-free search. PLoS ONE 2, e354. doi:10.1371/journal. pone. 0000354 .

Rodrigues, V. (1980). "Olfactory behavior of Drosophila melanogaster," in Development and Neurobiology of Drosophila, eds O. Siddiqi, P. Babu, L. M. Hall, and J. C. Hall (New York: Plenum), 361-371.

Rodrigues, V. (1988). Spatial coding of olfactory information in the antennal lobe of Drosophila melanogaster. Brain Res. 453, 299-307.

Rosenzweig, M., Brennan, K. M., Tayler, T. D., Phelps, P. O., Patapoutian, A., and Garrity, P. A. (2005). The Drosophila ortholog of vertebrate TRPA1 regulates thermotaxis. Genes Dev. 19, 419-424.

Sane, S.P. (2006). Induced airflow in flying insects I. A theoretical model of the induced flow. J. Exp. Biol. 209(Pt 1), 32-42.

Sato, K., Pellegrino, M., Nakagawa, T., Vosshall, L. B., and Touhara, K. (2008). Insect olfactory receptors are heteromeric ligand-gated ion channels. Nature 452, 1002-1006.

Schlief, M. L., and Wilson, R. I. (2007). Olfactory processing and behavior downstream from highly selective receptor neurons. Nat. Neurosci. 10, 623-630.

Schone, H. (1984). Spatial OrientationThe Spatial Control of Behavior in Animals and Man. Princeton, NJ: Princeton University Press.

Semmelhack, J. L., and Wang, J.W. (2009). Select Drosophila glomeruli mediate innate olfactory attraction and aversion. Nature 459, 218-223. 
Serway, C. N., Kaufman, R. R., Strauss, R., and de Belle, J. S. (2009). Mushroom bodies enhance initial motor activity in Drosophila. J. Neurogenet. 23, 173-184.

Shanbhag,S.R., Mueller,B., and Steinbrecht, R. A. (2000). Atlas of olfactory organs of Drosophila melanogaster 2. Internal organization and cellular architecture of olfactory sensilla. Arthropod. Struct. Dev. 29, 211-229.

Shang, Y., Claridge-Chang, A., Sjulson, L., Pypaert, M., and Miesenbock, G. (2007). Excitatory local circuits and their implications for olfactory processing in the fly antennal lobe. Cell 128, 601-612.

Silbering, A. F., and Galizia, C. G. (2007). Processing of odor mixtures in the Drosophila antennal lobe reveals both global inhibition and glomerulusspecific interactions. J. Neurosci. 27, 11966-11977.

Steck, K., Hansson, B., and Knaden, M. (2009). Smells like home: desert ants, Cataglyphis fortis, use olfactory landmarks to pinpoint the nest. Front. Zool. 6:5. doi:10.1186/1742-9994-6-5.

Steck, K., Knaden, M., and Hansson, B. S. (2010). Do desert ants smell the scenery in stereo? Anim. Behav. In Press, Corrected Proof. doi: 10.1016/ j.anbehav.2010.01.011.

Stephens, G. J., Johnson-Kerner, B., Bialek, W., and Ryu, W. S. (2008). Dimensionality and dynamics in the behavior of C. elegans. PLoS Comput. Biol. 4: e1000028. doi:10.1371/journal. pcbi. 1000028 .

Stocker, R. F. (2001). Drosophila as a focus in olfactory research: mapping of olfactory sensilla by fine structure, odor specificity, odorant receptor expression, and central connectivity. Microsc. Res. Tech. 55, 284-296.
Stocker, R. F., Lienhard, M. C., Borst, A. and Fischbach, K. F. (1990). Neuronal architecture of the antennal lobe in Drosophila melanogaster. Cell Tissue Res. 262, 9-34.

Stockinger, P., Kvitsiani, D., Rotkopf, S., Tirian, L., and Dickson, B. J. (2005). Neural circuitry that governs Drosophila male courtship behavior. Cell 121, 795-807.

Suh, G. S., Wong, A. M., Hergarden, A. C., Wang, J. W., Simon, A. F., Benzer, S., Axel, R., and Anderson, D. J. (2004). A single population of olfactory sensory neurons mediates an innate avoidance behaviour in Drosophila. Nature 431, 854-859.

Tanaka, N. K., Awasaki, T., Shimada, T., and Ito, K. (2004). Integration of chemosensory pathways in the Drosophila second-order olfactory centers. Curr. Biol. 14, 449-457.

Tanaka, N. K., Ito, K., and Stopfer, M. (2009). Odor-evoked neural oscillations in Drosophila are mediated by widely branching interneurons. $J$. Neurosci. 29, 8595-8603.

Tanaka, N. K., Tanimoto, H., and Ito, K. (2008). Neuronal assemblies of the Drosophila mushroom body. J. Comp. Neurol. 508, 711-755.

Tripathy, S., Staudacher, E., Peters, O., Kalwar, F., Hatfield, M., and Daly, K. (2010). Odors pulsed at wing beat frequencies are tracked by primary olfactory networks and enhance odor detection. Front. Cell. Neurosci. doi:10.3389/neuro.03.001.2010.

Turner, G. C., Bazhenov, M., and Laurent, G. (2008). Olfactory representations by Drosophila mushroom body neurons. J. Neurophysiol. 99, 734-746.

Turner, S. L., and Ray, A. (2009). Modification of $\mathrm{CO} 2$ avoidance behaviour in Drosophila by inhibitory odorants. Nature 461, 277-281.

Vergassola, M., Villermaux, E., and Shraiman, B. I. (2007). 'Infotaxis' as a strategy for searching without gradients. Nature 445, 406-409.

Vickers, N. J. (2000). Mechanisms of animal navigation in odor plumes. Biol. Bull. 198, 203-212.

Vosshall, L. B., Amrein, H., Morozov, P. S., Rzhetsky, A., and Axel, R. (1999). A spatial map of olfactory receptor expression in the Drosophila antenna. Cell 96, 725-736.

Vosshall, L. B., and Stocker, R. F. (2007). Molecular architecture of smell and taste in Drosophila. Annu. Rev. Neurosci. 30, 505-533.

Wang, J. W., Wong, A. M., Flores, J., Vosshall, L. B., and Axel, R. (2003). Two-photon calcium imaging reveals an odor-evoked map of activity in the fly brain. Cell 112, 271-282.

Welsh, J. H. (1937). The chemoreceptors of certain dipterous larvae. Science 85 , 430-431.

Wicher, D., Schafer, R., Bauernfeind, R., Stensmyr, M. C., Heller, R., Heinemann, S. H., and Hansson, B. S. (2008). Drosophila odorant receptors are both ligand-gated and cyclicnucleotide-activated cation channels. Nature 452, 1007-1011.

Wilson, R. I., and Laurent, G. (2005). Role of GABAergic inhibition in shaping odor-evoked spatiotemporal patterns in the Drosophila antennal lobe. J. Neurosci. 25, 9069-9079.

Wilson, R. I., and Mainen, Z. F. (2006). Early events in olfactory processing. Annu. Rev. Neurosci. 29, 163-201.

Wilson, R. I., Turner, G. C., and Laurent, G. (2004). Transformation of olfactory representations in the Drosophila antennal lobe. Science 303, 366-370.
Wu, Q., Wen, T., Lee, G., Park, J. H., Cai, H. N., and Shen, P. (2003). Developmental control of foraging and social behavior by the Drosophila neuropeptide Y-like system. Neuron 39, 147-161.

Yankov, E., Nakova, A., and Tzekov, R. (1999). Spectral sensitivity of the visual system as revealed by evoked potentials in normal and anomalous trichomats. Acta Physiol. Pharmacol. Bulg. 24, 91-100.

Yarali, A., Ehser, S., Hapil, F. Z., Huang, J., and Gerber, B. (2009). Odour intensity learning in fruit flies. Proc. Biol. Sci. 276, 3413-3420.

Zars, T., Fischer, M., Schulz, R., and Heisenberg, M. (2000). Localization of a short-term memory in Drosophila. Science 288, 672-675.

Conflict of Interest Statement: The authors declare that the research was conducted in the absence of any commercial or financial relationships that could be construed as a potential conflict of interest.

Received: 29 January 2010; paper pending published: 10 February 2010; accepted: 10 March 2010; published online: 31 March 2010.

Citation: Gomez-Marin A, Duistermars BJ, Frye MA and Louis M (2010) Mechanisms of odor-tracking: multiple sensors for enhanced perception and behavior. Front. Cell. Neurosci. 4:6. doi: 10.3389/fncel.2010.00006

Copyright (c) 2010 Gomez-Marin, Duistermars, Frye and Louis. This is an open-access article subject to an exclusive license agreement between the authors and the Frontiers Research Foundation, which permits unrestricted use, distribution, and reproduction in any medium, provided the original authors and source are credited. 